\title{
Multi scale characteristics of Antarctic temperature change in January from 1982 to 2020
}

\author{
Guo Huifang, Yin Fangfang*, Guangtian Cao \\ Zhejiang Tongji Vocational College of science and technology, Hangzhou, Zhejiang 311231, China
}

\begin{abstract}
Antarctica is a sensitive area of global temperature change. Analyzing the temperature change in Antarctica can provide a basis for the analysis of global climate change The shared data provided by the automatic weather station plan are analyzed, and the relatively complete January temperature of Antarctic 8905 station from 1981 to 2020 is selected for analysis. Using the multi-scale analysis function of wavelet analysis, Morlet wavelet function is selected to carry out continuous wavelet transform on the daily temperature data, and the real part, modulus square and variance of continuous wavelet change are analyzed. It is found that the daily temperature of Antarctica is There are 14, 27, 72, 268, 380 and 665 day main periods for temperature changes. The trend analysis of wavelet transform coefficients under each period shows that the Antarctic temperature is in a high temperature period of 14, 27, 72, 268, 380 and 665 day periods in the years after 2021. The maximum temperature in January in Antarctica has a 20-year main period, and the minimum temperature has a 14-year and 23-year main period, The average value has a main cycle of 6 years, 14 years and 24 years.
\end{abstract}

\section{Introduction}

The blue book on world climate change points out that from 1880 to 2012, the global average surface temperature increased by about $0.85^{\circ}$. In the next 100 years, the global average surface temperature will increase by 1.4 to 5.8 degrees Celsius, becoming the fastest warming period in recent 10000 years. Global warming has led to the rise of sea level and the frequent occurrence of various climate disasters in land areas. As an important part of the earth system, the polar climate system is the most sensitive area to climate warming. Analyzing the law of temperature change in the polar region is helpful to provide a reference basis for global research on the characteristics of climate change. At present, many scholars at home and abroad have carried out research and Analysis on temperature changes in Antarctica. Nicolas et al. [1,2] studied extreme high temperature events in Antarctica through era interim reanalysis data. Li Jingfeng [3] and others analyzed the characteristics of temperature in the north of the Antarctic Peninsula and its relationship with sea ice. Liu $\mathrm{Yu}$ [4] analyzed the impact of tropical intraseasonal oscillation on Antarctic temperature change. Wang Yicheng et al. [5] used polar WRF to analyze extreme low temperature events in Antarctica. Ma Qiang et al. [6] analyzed the variation characteristics of temperature, wind and precipitation at the Great Wall Station in Antarctica. Using the shared data provided by the automatic weather station program of the University of Wisconsin (Madison), the temperature data of 8905 station is selected for analysis by analyzing the data of all observation stations.
The temperature observation data of the station is relatively sound, and the observation data are from 1982 to 2020 . However, some monthly data are missing. This time, relatively continuous January temperature data are selected for analysis to issue the internal laws of Antarctic temperature change and find out its internal scale and change trend at each scale. Wavelet analysis is a good multi-scale analysis method. It can find the internal laws of data through Fourier change. It is also known as the "magnifying glass" of data analysis.

\section{Analysis of Antarctic temperature change based on CWT}

If the Antarctic temperature data sequence is $f(t)$, its continuous wavelet transform CWT is $[7,8]$ :

$W_{f}(a, b)=|a|^{-1 / 2} \int_{t=-\infty}^{\infty} f(t) \Psi\left(\frac{t-b}{a}\right) d t$

Among them: $\mathrm{W}_{\mathrm{f}}(\mathrm{a}, \mathrm{b})$ is the wavelet transform coefficient, $\Psi_{\mathrm{a}, \mathrm{b}}(\mathrm{t})$ is the continuous wavelet function, which is also called the basic wavelet. It has the characteristics of small energy, small shape, oscillation in time domain, good vanishing moment and good self phasing. Namely:

$$
\int_{-\infty}^{\infty}\left|\Psi_{a, b}(t)\right|^{2} d t=\int_{-\infty}^{\infty}|\Psi(t)|^{2} d t=1
$$

\footnotetext{
*Corresponding author: 61681034@qq.com
} 


$$
\begin{gathered}
\int_{-\infty}^{\infty} \Psi_{a, b}(t) d t=\int_{-\infty}^{\infty} \Psi(t) d t=0 \\
\int_{-\infty}^{\infty} t^{p} \Psi(t) d t=0
\end{gathered}
$$

The Antarctic temperature signal can be changed and reduced by adjusting the size of a and $\mathrm{B}$. If you need to analyze the signal details, you can adjust a to reduce, $\Psi_{\mathrm{a}, \mathrm{b}}(\mathrm{t})$ ' $\mathrm{s}$ value and shrink in the direction of the time axis, so as to obtain high-frequency information. If it is necessary to analyze the general picture of wavelet, a can be increased, which $\Psi_{\mathrm{a}, \mathrm{b}}(\mathrm{t})$ extends in the direction of time axis, so as to obtain the low-frequency information contained in the signal.

In practical application, the continuous wavelet function must meet the admissibility condition. At present, the commonly used Morlet function is the wavelet function that can meet this condition. Morlet wavelet is a single frequency complex sinusoidal function. The expression is:

$$
\Psi(t)=e^{i w_{0} t} e^{-t^{2} / 2}
$$

Morlet wavelet function is used to continue the multiscale analysis of the Antarctic temperature change.

\section{Multiscale characteristic analysis of Antarctic temperature}

The analysis data is the temperature data of Antarctic 8905 station from January 1981 to 2020 . The observation data is measured once every 3 hours, but some of them are missing. The average data of the first three hours and the last three hours are used to analyze the missing data. The data of three years are missing, and the data of these three years are not involved in the analysis. The data analyzed in this time are daily temperature data series, annual maximum temperature data series, annual minimum temperature series and annual average temperature series. The above Morlet wavelet function is selected to carry out continuous wavelet change on the Antarctic temperature change, and the existing law of Antarctic temperature change is found by calculating the main eigenvalues of wavelet transform coefficients.

\subsection{Multiscale analysis of daily temperature in Antarctica}

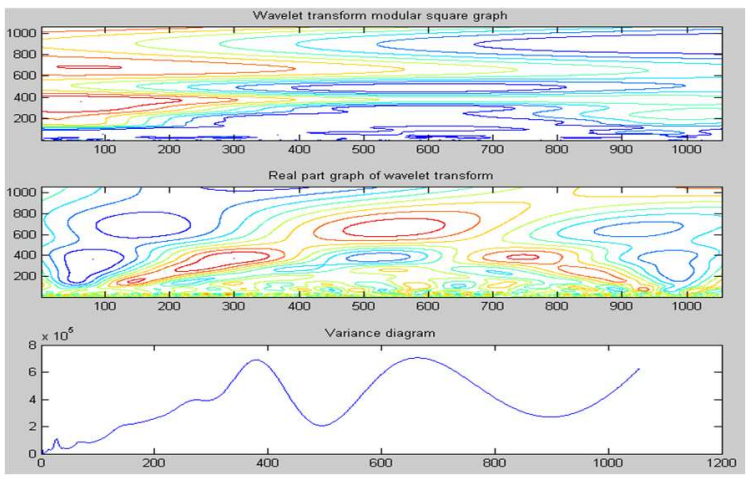

Fig. 1 Multiscale analysis of daily temperature in Antarctica
By analyzing the modulus square, real part and variance of the wavelet transform of daily temperature in Antarctica, and enlarging the turning point, it is found that there are 14, 27, 72, 268, 380 and 665 day main cycles in the daily temperature change series in Antarctica in January. By analyzing the real part change process of wavelet transform at each main scale, the change process of temperature series at various scales can be obtained.

20

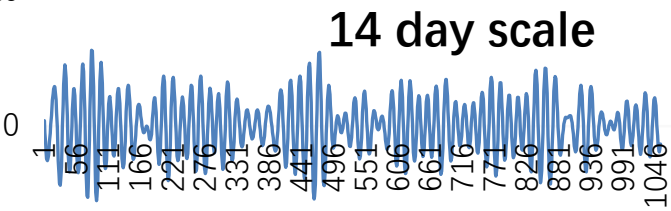

$-20$

Fig. 2 Analysis of Antarctic temperature change on 14 day scale

In the 14 day scale, the periodic change of temperature is more intense. According to the analysis above, the temperature of Antarctica in January 2021 is in a high temperature period on the 14 day scale.

50

\section{7 day scale}

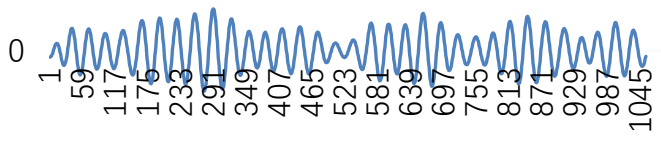

Fig. 3 Analysis of Antarctic temperature change on 27 day scale

In the 27 day scale, the periodic change of temperature is more intense. According to the analysis of the above figure, the temperature in January 2021 in Antarctica is in a high temperature period on the scale of 27 days.

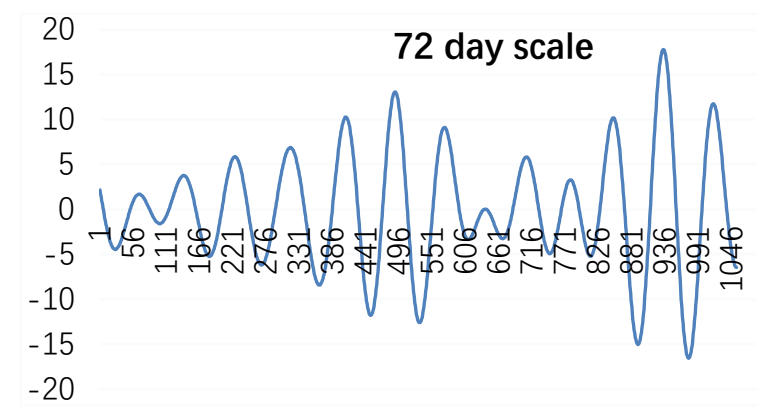

Fig. 4 Analysis of Antarctic temperature change on 72 day scale

It can be seen from the above figure. In January 2021, the temperature in Antarctica is in the low temperature period of 72 day scale. In January 2022, the temperature in Antarctica is in the high temperature period of 72 day scale. 


\section{0

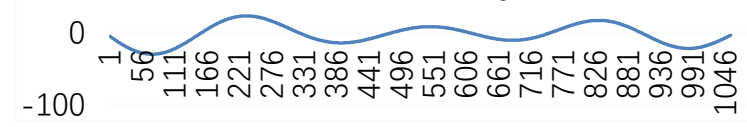

Fig. 5 Analysis of Antarctic temperature change on 268 day scale

It can be seen from the above figure that the temperature of Antarctica in January in 2021, 2022, 2023 and 2024 is in the period of high temperature on the scale of 268 days. After 2006, the turning years of temperature from low to high are 2020 and 2009 . The turning years of temperature from high to low are 2015 and 2006.

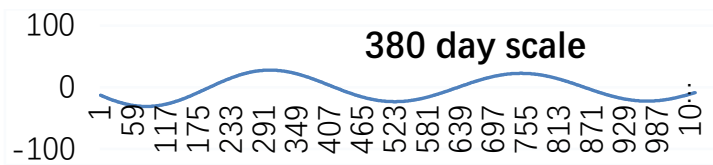

Fig. 6 Analysis of Antarctic temperature change on 380 day scale

It can be seen from the above figure that the temperature in January of Antarctica in 2021, 2022, 2023, 2024, 2025 and 2026 is in a high temperature period under the 380 day scale. The turning years of temperature from low to high after 2006 are 2020 and 2006. The turning year of temperature from high to low is 2013.

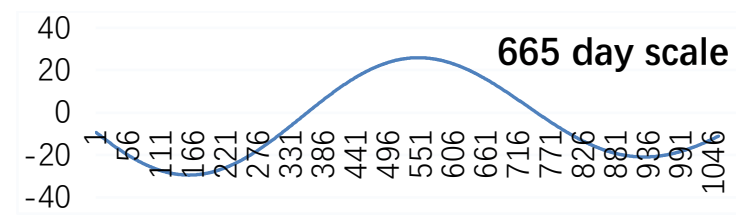

Fig. 7 Analysis of Antarctic temperature change on 665 day scale

It can be seen from the above figure that the temperature of Antarctica in January from 2022 to 2029 is in a high temperature period on the 665 day scale. After 2006, the turning year of temperature from low to high is 2021; The turning year of temperature from high to low is 2010.

\subsection{Multi scale analysis of temperature variation in Antarctica in January}

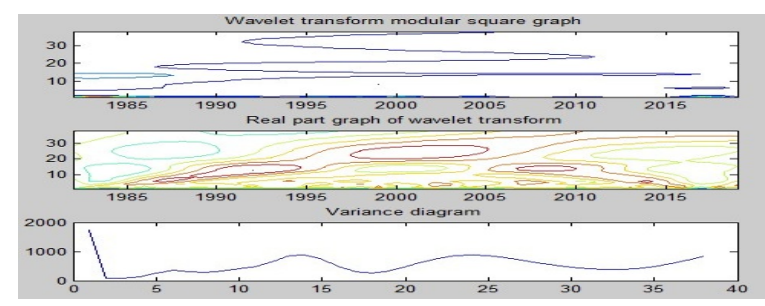

Fig. 8 Multi scale characteristic analysis of temperature change in Antarctica in January

The modulus square, real part and variance of the wavelet transform of the average temperature in January in Antarctica are analyzed, and the turning point is enlarged.
It is found that it has a main cycle of 6 years, 14 years and 24 years.

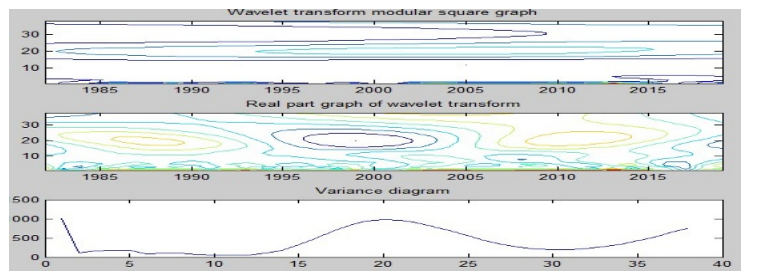

Fig. 9 Multi scale characteristic analysis of temperature change in Antarctica in January

The modulus square, real part and variance of the wavelet transform of the maximum temperature in January in Antarctica are analyzed, and the turning point is enlarged. It is found that there is a main period of 20 years.

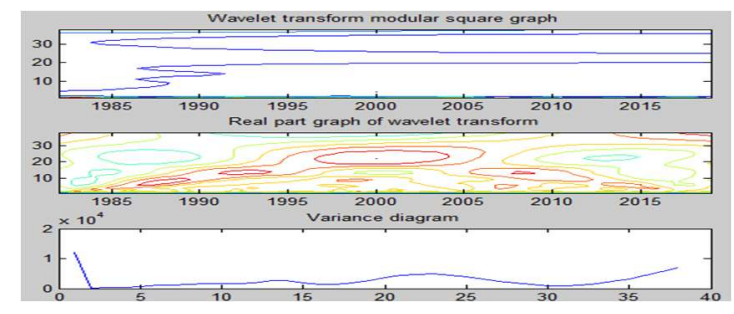

Fig. 10 Multi scale characteristic analysis of temperature change in Antarctica in January

The modulus square, real part and variance of the wavelet transform of the maximum temperature in January in Antarctica are analyzed, and the turning point is enlarged. It is found that there are 14-year and 23-year main cycles.

\section{Conclusion}

Antarctica is a sensitive area of global temperature change. Analyzing the temperature change in Antarctica can provide a basis for the analysis of global climate change. Using the shared data provided by the automatic weather station plan of the University of Wisconsin (Madison), the temperature data of 8905 station in January are selected for analysis. Using the multi-scale analysis method of continuous wavelet transform, the main periods of daily temperature change in Antarctica are analyzed, which are $14,27,72,268,380$ and 665 days. The trend of wavelet transform coefficients in each period is analyzed. It is found that the Antarctic temperature is in the high temperature period of 14, 27, 72, 268, 380 and 665 days in the years after 2021. The maximum temperature in January in Antarctica has a main cycle of 20 years, the minimum has a main cycle of 14 and 23 years, and the average has a main cycle of 6,14 and 24 years. In the next few years, the global temperature will be in a period of high temperature. All climate change related units should take response measures such as energy conservation and emission reduction and green environmental protection to reduce carbon dioxide emissions, so as to reduce the impact of human activities on temperature and slow down the rising trend of global temperature. 


\section{Acknowledgments}

This work was financially supported by Water conservancy science and technology plan project of Zhejiang Province (Project No.RC2008) fund.

\section{References}

1. Nicolas J P, Vogelmann A M, Scott R C, et al. January 2016 extensive summer melt in West Antarctica favoured by strong El Niño[J]. Nature Communications, 2017, 8: 15799.

2. de Los Milagros Skansi M, King J, Lazzara M A, et al. Evalu-ating the highest temperature extremes in the antarctic[J]. Eos, 2017, 98(5): 18 - 23.

3. Li Jingfeng, Bian Lingen, Duan Juqi, Ding Zhuoming, Wei Qinwu, Lu Changgui. Temperature characteristics in the northern Antarctic Peninsula and their relationship with sea ice $[\mathrm{J}]$. Meteorological science and technology, 2016,44 (05): 763-768

4. Liu Yu. Influence of tropical intraseasonal oscillation on Antarctic temperature change [D]. Nanjing University of information engineering, 2019

5. Wang Yicheng, Xie Aihong, Ding Minghu, Xiao cunde. Analysis of extreme low temperature event in Antarctic Dome A based on polar WRF [J]. Glacier and frozen soil, 2020,42 (03): 727-733

6. Ma Qiang, Wang Yating, Zhang Cui, he Yun, Guo Guangyuan. Analysis on variation characteristics of temperature, wind and precipitation at the Great Wall Station in Antarctica [J]. Shaanxi meteorology, 2016 (01): $25-28$

7. Cui Jintai. Introduction to wavelet analysis [M]. Xi'an: Xi'an Jiaotong University Press, 1995:1 20

8. Ma Xixia, Hu Tiecheng. Annual runoff prediction of reservoir based on ANFIS [J]. Journal of hydropower, 2008 (4): 33-37 